\title{
Overview of the ATLAS Insertable B-Layer (IBL) Project
}

\author{
M. A. Kagan* on behalf of the ATLAS Collaboration \\ $S L A C$, \\ Menlo Park, CA 94025, USA \\ *E-mail: makagan@slac.stanford.edu \\ www.slac.stanford.edu
}

\begin{abstract}
The first upgrade for the Pixel Detector will be a new pixel layer which is currently under construction and will be installed during the first shutdown of the LHC machine, in 2013-14. The new detector, called the Insertable B-layer (IBL), will be installed between the existing Pixel Detector and a new, smaller radius beam-pipe. Two different silicon sensor technologies, planar n-in-n and $3 \mathrm{D}$, will be used, connected with the new generation 130nm IBM CMOS FE-I4 readout chip via solder bump-bonds. A production quality control test bench was set up in the ATLAS inner detector assembly clean room to verify and rate the performance of the detector elements before integration around the beampipe. An overview of the IBL project, of the module design, the qualification for these sensor technologies, the integration quality control setups and recent results in the construction of this full scale new concept detector is discussed.
\end{abstract}

Keywords: ATLAS; IBL; Pixel Detectors; ATLAS Upgrade.

\section{Introduction}

During 2013-14, the Large Hadron Collider (LHC) ${ }^{1}$ will upgrade to the design energy of 13 to $14 \mathrm{TeV}$ and instantaneous luminosities of $10^{34} \mathrm{~cm}^{-2} \mathrm{~s}^{-1}$. At these energies and luminosities, the high number of interactions per bunch crossing can cause significant degradation of the performance of tracking, vertexing, and b-tagging algorithms of the ATLAS detector. ${ }^{2}$ In addition, ATLAS is expected to acquire $300 \mathrm{fb}^{-1}$ of data, for which the expected fluence of $50 \mathrm{MRad}$ ionizing dose and $10^{15} \mathrm{n}_{\mathrm{eq}} / \mathrm{cm}^{2}$ non-ionizing dose will lead to significant radiation damage to the Pixel Detector. ${ }^{3}$ To mitigate these effects, a new fourth layer of pixel modules, the Insertable B-Layer (IBL), ${ }^{4}$ will be inserted between the current Pixel Detector and a new smaller radius beam-pipe. The IBL sensors will be located at a radius of $33 \mathrm{~mm}$ from the beam-axis and cover $|\eta|<2.9$. Due to the close proximity to the interaction point, the IBL must withstand 250 MRad of ionizing 
dose and $5 \times 10^{15} \mathrm{n}_{\mathrm{eq}} / \mathrm{cm}^{2}$ non-ionizing dose over the $300 \mathrm{fb}^{-1}$ lifetime, and maintain a hit efficiency of $>97 \%$. In addition, the front-end electronics must cope with high radiation levels and track occupancies, and must have a high active fraction due to the tight $(2 \mathrm{~cm})$ space constraints of the IBL making shingling of sensors along the $z$-direction impossible. To meet these requirements, new sensors in conjunction with a new FE chip, the FE-I4, with reduced pixel size and low material budget have been developed.

\section{IBL Geometry and Layout}

The IBL consists of 14 staves arranged along the beam line and around the beam-pipe with a $14^{\circ}$ incline allowing for an azimuthal overlap of the staves to ensure complete coverage in $\phi$ and to compensate for the Lorentz angle in the $2 \mathrm{~T}$ solenoidal magnetic field of the ATLAS inner detector. The IBL staves are mounted on an Inner Positioning Tube (IPT), which surrounds a new smaller radius $(\sim 24 \mathrm{~cm})$ beam-pipe, and inside of the IBL Support Tube (IST). The layout can be found in Figure 1.
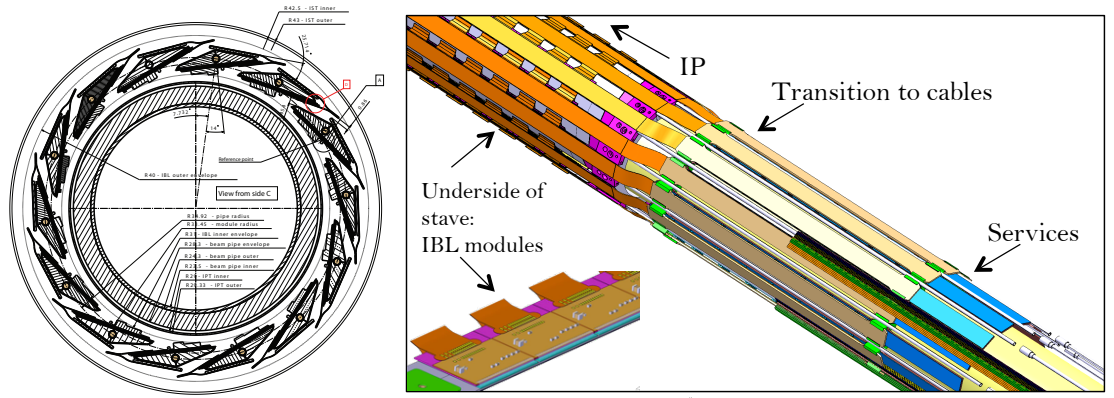

Fig. 1. Left: Cross-section view $(r-\phi)$ of the IBL. Right: Longitudinal view of IBL mounted on beam-pipe with connection to services

Each IBL stave is $64 \mathrm{~cm}$ long and holds 32 FE-I4 chips which are bump bonded onto silicon sensors. Two kinds of sensors are used: the 12 central modules are Double-Chip (DC) planar sensors with two FE-I4s per sensor, and the outer-most 4 modules on each side are Single-Chip (SC) 3D sensors with one FE-I4 per sensor. IBL modules are constructed by gluing a flexible $\mathrm{PCB}$, the module flex, on each sensor and wire bonding the module flex to the front-end chip, allowing the routing of data and services to the FE-I4s.

The IBL staves are the mechanical support structure for the sensors and 
electronics, and are built from a stiff K13C/RS3 ${ }^{\mathrm{a}}$ carbon fibre material filled with carbon foam ${ }^{\mathrm{b}}$ (serving as the heat conductor) surrounding a titanium cooling pipe. The material budget of the staves is $\leq 0.6 \% X_{0}$. In order to minimize radiation damage to the sensors, the IBL will be operated at $\leq-20^{\circ} \mathrm{C}$ using evaporative $\mathrm{CO}_{2}$ cooling. The FE-I4 side of the modules is glued onto the stave face-plate, and the module flex is wire-bonded to a flexible PCB bus (glued onto the side of the stave) via flex wings (one per front-end) to provide connection to services from outside of the stave.

The IBL planar sensors ${ }^{5}$ use an $\mathrm{n}^{+}$-in-n design and are manufactured by $\mathrm{CiS}^{\mathrm{c}}$. The sensors are $200 \mu \mathrm{m}$ thick. To minimize the inactive edge of the sensors to approximately $200 \mu \mathrm{m}$, the structure of 13 guard rings is shifted into the active pixel region under elongated $500 \mu \mathrm{m}$ wide edge pixels.

The IBL 3D double-sided p-type sensors ${ }^{6}$ are $230 \mu \mathrm{m}$ thick with two $n^{+}$electrodes per pixel surrounded by six $p^{+}$biasing electrodes. The distance between electrodes in $67 \mu \mathrm{m}$ and the inactive edge of the sensor is minimized to approximately $200 \mu \mathrm{m}$ using a guard fence design. The small depletion width between electrodes leads to faster charge collection, lower charge trapping probability, and lower bias voltages than planar sensors. The sensors were produced by $\mathrm{FBK}^{\mathrm{d}}$ and $\mathrm{CNM}^{\mathrm{e}}$.

The new FE-I4 front-end chip ${ }^{7}$ uses $130 \mathrm{~nm}$ feature size CMOS process to cope with the higher hit occupancy and radiation levels expected for the IBL. The FE-I4 has an active area of $16.8 \mathrm{~mm} \times 20 \mathrm{~mm}$ and contains the readout for 26880 hybrid pixels of size $50 \mu \mathrm{m} \times 250 \mu \mathrm{m}$ arranged in 80 columns and 336 rows which are connected to the sensors by bump bonding each individual pixel cell. Each pixel cell contains an amplification stage, with adjustable shaping, followed by a discriminator, with adjustable threshold, which allows the measurement of the discriminator firing time and the time-over-threshold (ToT). The pixel array is subdivided into $2 \times 2$ pixel regions which share a common Pixel Digital Region (PDR) holding memory, hit logic, and trigger processing. The PDR allows for high occupancy running, lower power consumption, and improved active areas. As such, the FE-I4 has a maximum trigger rate of $200 \mathrm{kHz}$ and a hit inefficiency of $<1 \%$ at occupancies of $400 \mathrm{MHz} / \mathrm{cm}^{2}$.

The performance of unirradiated and irradiated (with fluences of $\geq$

aProvided by TenCare, USA, http://www.tencare.com

${ }^{b}$ Provided by Allcomp Inc., USA, http://www.allcomp.com

${ }^{\mathrm{c}} \mathrm{CiS}$, Erfurt, Germany, http://www.cismst.org

${ }^{\mathrm{d}}$ Fondazione Bruno Kessler, Trento, Italy, http://www.fbk.eu

${ }^{\mathrm{e}}$ Centro Nacional de Microelectronica, Barcelona, http://www.cnm.es 
$\left.5 \times 10^{15} \mathrm{n}_{e q} / \mathrm{cm}^{2}\right)$ IBL modules has been evaluated in test-beams ${ }^{8}$ at DESY ( $4 \mathrm{GeV}$ positrons) and at CERN (120 GeV pions) using the EUDET ${ }^{9}$ telescope to reconstruct the beam trajectories with high resolution as the main external reference system. Cell efficiencies of $98 \%$ for both planar and 3D sensors with tracks at a $0^{\circ}$ incidence, and efficiencies of $97.7 \%$ for planar and $99 \%$ for $3 \mathrm{D}$ sensors with tracks at a $15^{\circ}$ incidence were observed. The bias voltages needed for full depletion were $V_{D}=-1000(-160) \mathrm{V}$ for planar (3D) sensors. The size of the inactive edge determined from edge pixel efficiencies was found to be $200 \mu \mathrm{m}$ wide for both planar and 3D sensors, thus showing that slim-edges have been achieved.

\section{Stave Testing}

A dedicated testing and Quality Assurance (QA) stand has been setup at CERN for the simultaneous qualification at warm $\left(10^{\circ} \mathrm{C}\right)$ and cold $\left(-20^{\circ} \mathrm{C}\right)$ temperatures of two staves in an environmentally controlled box equipped with a $\mathrm{CO}_{2}$ cooling system. The test-stand contains a linear motion device for aligning ${ }^{90} \mathrm{Sr}$ and ${ }^{241} \mathrm{Am}$ sources above modules, and has scintillators above and below the box for triggering on cosmic events. Each stave is systematically tested to check the modules break-down voltages and I-V curves, signal transmission, FE-I4 power consumption, behavior at high thresholds $\left(3000 e^{-}\right)$and low threshold $\left(1500 e^{-}\right)$, noise levels, cross-talk, and for low numbers $(<0.2 \%)$ of pixel failures. To ensure the modules work in realistic data taking conditions, both cosmic and radioactive source data runs are taken. Figure 2 shows the stability of the $-20^{\circ} \mathrm{C}$ threshold (noise) for several tunings for each module on a stave in the left (middle) figure, and the right figure shows the occupancy of 4 modules irradiated by a ${ }^{90} \mathrm{Sr}$ source and showing $<0.1 \%$ bad pixels.
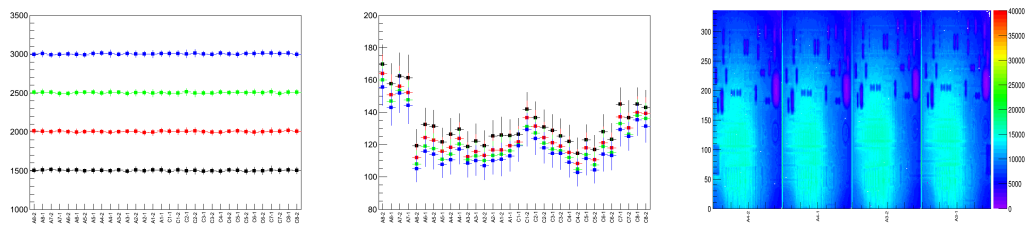

Fig. 2. Left (Middle): Threshold (noise) per module. Right: ${ }^{90} \mathrm{Sr}$ occupancy on modules. 


\section{Construction, Integration, and Installation Status}

Construction of the IBL is currently underway. Module production, including bump-bonding of the FE-I4's to the sensors, the gluing of the module flex to the sensor back-side, and wire bonding of the module flex to the FE-I4's, will finish in October 2013, with a total of 168 DC and 112 SC high quality modules needed. High quality modules are loaded onto staves and connected to the stave flex via the flex wings, and subjected to indepth testing. Currently 9 staves have been produced with 6 fully tested and qualified. The first production staves have been of exceptional quality, with stable tunings, low noise levels, and $<0.1 \%$ of bad pixels.

The integration of the IBL then proceeds though brazing of the titanium cooling pipe extension to the stave, mounting of the stave around the new beam-pipe using the multi-purpose-container (MPC) loading device, connection of services, and final full retesting on the surface. The stave integration onto the will begin October 2013 and finish by early 2014, to allow for IBL installation into the ATLAS cavern in early 2014.

\section{Summary and Outlook}

The first upgrade of the ATLAS pixel detector, the Insertable B-Layer (IBL), is a new $4^{\text {th }}$ layer of pixels that will be installed on a new smaller radius beam-pipe during the 2013-14 shutdown of the LHC. The IBL will use slim-edge planar and 3D sensors with a new front-end chip, the FE-I4, to cope with the expected high occupancies and radiation levels. Test-beams show excellent results from both sensor designs, including a $200 \mu \mathrm{m}$ active edge. The first IBL staves have shown excellent behavior on a dedicated QA test-stand at CERN. Stave production will finish by the end of 2013, and integration of the staves onto the beam-pipe should finish by early 2014 . The IBL will be installed into the ATLAS experiment in early 2014 .

\section{References}

1. L. Evans and P. Bryant, JINST 3, S08001 (2008).

2. ATLAS Collaboration, JINST 3, S08003 (2008).

3. G. Aad et al., JINST 3, P07007 (2008).

4. ATLAS Collaboration, CERN-LHCC-2010-013, 12 (2010).

5. C. Gossling et al., NIM A. 650 (2011).

6. C. Da Via et al., NIM A. 604 (2009).

7. V. Zivkovic et al., JINST 7, C02050 (2012).

8. ATLAS IBL Collaboration, JINST 7, P11010 (2012).

9. EUDET-JRA1 Collaboration, A.Bulgheroni et al., NIM A. 623 (2010). 